\title{
Is a sequence of tests during urethral pressure profilometry correlated with symptoms assessment in women?
}

Françoise A. Valentini, Gilberte Robain, Brigitte G. Marti

ER6 - Université Pierre et Marie Curie (Paris 06) (FAV, GR); Service de Médecine Physique et Rééducation (Neurologie), Hôpital Rothschild (FAV, GR) and Service de Rééducation, Hôpital Saint Antoine (BGM), Paris, France

\section{ABSTRACT}

Introduction: Our purpose was, applying a strictly defined protocol for urethral profilometry, 1) to test the repeatability of same session rest maximum urethral closure pressure (MUCP) and 2) to search for correlation between women complaint and the changes in MUCP value (rest and dynamic tests).

Materials and Methods: A population of 140 consecutive women referred for evaluation of lower urinary tract dysfunction was stratified in 4 groups according with the urinary symptoms: stress, urge, mixed incontinence and continent and in each group in 3 age groups (young, middle age and old). The sequence of tests recorded in supine position was: urethral pressure profile at rest bladder empty, after bladder filling at $250 \mathrm{~mL}$ (reference test), stress profile, fatigability (before (rest) and after 10 successive strong coughs), then in standing position.

Results: In all groups, there was no significant difference between the two MUCP values at rest bladder filled. In the three incontinent groups, MUCP was higher bladder empty than bladder filled ( $p<0.05$ ) except in the young sub-group. Stress incontinence led to significant decrease of MUCP during dynamic tests in the young group. MUCP was not modified after fatigability test in women with urge complaint whatever age.

Conclusion: When recorded following a strictly defined protocol, MUCP at rest bladder filled has a good repeatability in individual. However a complex sequence of tests during urethral pressure profilometry remains discussed in middle-age and old age-groups, it allows specifying the stress component of incontinence in young women and the urgency component in all age-groups.

\section{ARTICLE INFO}

\section{Key words:}

Urethral Stricture; Pressure,

Women; Urethral Diseases

Int Braz J Urol. 2012; 38: 809-17

Submitted for publication:

January 23, 2012

Accepted after revision:

July 06, 2012

\section{INTRODUCTION}

Controversies about the role of urethral pressure profilometry (UPP) in clinical practice (1-3) are mainly based on the reproducibility of the measurements and on the lack of standardization (4).

Other main controversies are related to the predictive value of the preoperative maximum urethral closure pressure (MUCP) and the success of the surgery of stress incontinence (59) and the postoperative quality of life (10). Conversely, De Lancey (11) found that MUCP was the most characteristic parameter of stress incontinence.

In our urodynamic laboratory, we have defined a strict protocol for UPP which is routinely applied in order to rule out many causes of variability. It consists of a well-defined sequence 
of tests. UPP is always performed by the same two nurses and the tests always performed following the same sequence. If it is not observed any alteration of the MUCP value at rest, we search for a correlation between the patient complaints and MUCP value changes following dynamic tests.

Is the MUCP value at rest reproducible during a session whatever the patient complaints are? Do the changes in MUCP values during different dynamic tests provide information on the lower urinary tract dysfunction? The aim of this retrospective study was to try to give an answer to these questions.

\section{MATERIALS AND METHODS}

\section{Population}

The studied population consisted of 140 consecutive women without neurological disease or/and pelvic organ prolapse of grade $>2$ (mean age $56.8 \pm 15.5$ years [21-90 years]) referred for evaluation of lower urinary tract dysfunction (June 2007 to March 2009). Each woman completed a 3-day frequency-volume chart and the ICIQ-UI-SF before the urodynamic testing; this allowed a stratification of the population in four groups according with the symptoms: continent, with stress incontinence, urge incontinence, or mixed incontinence. The continent women complained of urgency or pain.

Stress incontinence is defined as involuntary leakage of urine following effort or exertion, including sneezing or coughing; urge incontinence is defined as the involuntary leakage accompanied by or immediately preceded by urgency, and mixed incontinence as the involuntary leakage associated with stress and urge conditions (12). A second stratification was made according to age: less than 45 years old (before menopause), 45 to 65 years old (menopausal transition) and above 65 years old (long term menopause). In France, the mean age for menopause is 50 years.

This study was conducted in accordance with the Declaration of Helsinki. According to the local practice of our Ethics Committee, there is no formal Institutional Review Board approval required for retrospective studies performed with the usual procedure of investigation.

\section{Urodynamic session}

A urodynamic session included the following tests: 1) free uroflowmetry at arrival; 2) UPP with empty bladder (supine position) before filling cystometry and pressure flow study (seating); 3) UPP bladder filled with $250 \mathrm{~mL}$ (or less, in cases of detrusor overactivity, at normal desire to void) with saline at room temperature (supine position except for standing recording) and 4) free uroflowmetry. Cystometry (filling rate $50 \mathrm{~mL} / \mathrm{min}$.) and UPP were performed using a $7 \mathrm{~F}$ triple lumen water perfusion catheter. Pressures were zeroed to atmosphere with the transducers placed at the level of the upper edge of the pubic symphysis. All urodynamic studies were performed using a Dorado ${ }^{\circledR}$ unit from Laborie and conducted by two nurses who applied our protocol.

Urodynamic testing was performed according with Good Urodynamic Practices (13) and, during cystometry, women were asked to cough during filling at $100 \mathrm{~mL}$ intervals to ensure that pressure signals responded equally.

Urethral protocol: For UPP the puller speed was set to $1 \mathrm{~mm} / \mathrm{s}$ except for standing recording when the catheter was pulled manually (14). The distal eye-hole was kept in the lateral position and the catheter position observed during the test to avoid change in orientation. The sequence of test was: (1) UPP at rest; (2) Kegel manoeuvre at MUCP; (3) UPP with 3 to 5 successive coughs (stress profile); (4) VLPP; (5) UPP before 10 successive strong coughs and (5') after (fatigability test), then (6) UPP was recorded in standing position.

\section{Recordings}

All MUCP values were reviewed independently by 2 investigators. Good agreement occurred in up to $88 \%$ of the files. In the remaining $12 \%$, an additional interpretation was made jointly to reach a single conclusion.

\section{Comparisons}

MUCP value at rest (empty bladder) (P1) was compared with (P0), (P3), (P5), (P5') and (P6) according with the continence status in the whole population and in the age groups. The relevant MUCP value (P3) was the value obtained after ma- 
nual smoothing of the stress profile in order to exclude the spikes due to cough efforts.

\section{Statistical analysis}

The numerical data are described by means and standard deviation and categorical data as percentages. The Wilcoxon signed rank test was used for comparison of related samples, analysis of variance and the Chi 2 test to compare unrelated samples. Statistical analysis was performed using SAS, version 5.0 (SAS Institute, Inc., Cary, $\mathrm{NC}$ ). All statistical results were considered significant at $\mathrm{p}<0.05$.

\section{Reproducibility}

The Bland-Altman plot (15) (difference vs. mean plot) was used to show the agreement between the two measurements of MUCP during resting profile (empty bladder).

\section{RESULTS}

Demographic data showed that there was no significant difference in age and in percentage of menopause between the 4 symptoms groups; previous pelvic surgery was more frequent in the groups with urge or mixed incontinence (Table-1). There was no significant difference neither in subjective symptoms appraisal in incontinent groups nor in VLPP between stress and mixed incontinence groups (Table-1). The motive for referring was not significantly different in the 3 age-groups.

\section{Analysis of all subjects (Table-2)}

Whatever the continence status, there was a good reproducibility during a session of the resting MUCP (empty bladder) (P1 and P5) (t-test and Bland-Altman plots, Figure-1).

In incontinent women, MUCP with filled bladder (P1) decreased significantly when compared to MUCP with empty bladder (P0). MUCP of stress profile ( $(\mathrm{P} 3)$, after fatigability test ( $\left.\mathrm{P}^{\prime}\right)$ and standing (P6) were significantly lower than MUCP at rest in women with stress or mixed incontinence. We observed no difference between MUCP values during dynamic testing (P3, P5'and P6) and the resting value $(\mathrm{P} 1)$ in women with urge incontinence.

In continent women, only two significant decreases of MUCP were observed: during the stress profile $(p=0.0012)$ and after the fatigability test $(p=0.0005)$.

Table 1 - Patients' characteristics. Data are given as mean \pm standard deviation.

\begin{tabular}{lcccc}
\hline Group & Continent & Stress Inc. & Urge Inc. & Mixed Inc. \\
\hline No women & 22 & 34 & 40 & 44 \\
Age & $54 \pm 15 \mathrm{y}$ & $55 \pm 14 \mathrm{y}$ & $57 \pm 15 \mathrm{y}$ & $59 \pm 17 \mathrm{y}$ \\
Parity & $2.2 \pm 1.5$ & $1.6 \pm 1.2$ & $1.6 \pm 1.3$ & $1.9 \pm 1.4$ \\
$\%$ menopausal & 59 & 65 & 65 & 71 \\
\% previous pelvic surgery & 33 & 44 & 61 & 55 \\
VAS / ICIQ-UI SF & $5 \pm 3$ & $11.2 \pm 5.4$ & $13.1 \pm 3.8$ & $13.0 \pm 4.3$ \\
pves (cm Ho) during Valsalva maneuver & $74 \pm 25$ & $61 \pm 21$ & $70 \pm 26$ & $70 \pm 25$ \\
VLPP (No patients) $\mathrm{cm} \mathrm{H}_{2} \mathrm{O}$ & 85 & $70 \pm 23$ & $52 \pm 10$ & $77 \pm 27$ \\
& $(\mathrm{No}=1)$ & $(\mathrm{No}=17)$ & (No $=5)$ & (No $=17$ ) \\
\hline
\end{tabular}

Abbreviation: Inc. Incontinence 
Analysis per age-group (Tables 3, 4 and 5)

In all continence sub-groups, as in the whole population, there was no significant difference between the MUCP measurements (P1 and P5) at rest (empty bladder).

In the young group (Table-3), MUCP decreased significantly during the stress profile in the SUI sub-group ( $p=0.0028)$ and after the fatigability test for women with SUI ( $p=0.0277)$ or mixed incontinence ( $\mathrm{p}=0.0330)$ (that last type of incontinence implying a stress component). There was a good reproducibility of MUCP during all tests for both continent and urge incontinence subgroups.

In the middle age group (Table-4), changes in MUCP were significant with filled bladder (P1) vs. empty bladder (P0) except in the continent subgroup, during stress profile (P3) in all subgroups, after fatigability (P5') in the SUI and mixed incontinence subgroup, and only in the SUI group in standing position (P6).

In the oldest group (Table-5) with SUI, the change in MUCP was significant with filled bladder (P1) vs. empty bladder (P0) $(\mathrm{p}=0.0374)$ and during the stress profile $(\mathrm{P} 3)(\mathrm{p}=0.0103)$. Changes in MUCP were observed in all tests for the mixed incontinence subgroup but only with empty bladder $(\mathrm{PO})(\mathrm{p}=0.0054)$ and standing (P6) $(\mathrm{p}=0.0015)$ in the urge subgroup. In the continent subgroup, MUCP decreased only after fatigability test (P5').

Figure 1 - Assessment of measurements reproducibility. Bland and Altman plots (15) for MUCP value at rest (empty bladder) in supine position. (P1): first measurement bladder filled, (P5): before fatigability test. (a) continent women, (b) women with stress incontinence complaint, (c) women with mixed incontinence complaint, (d) women with urge incontinence complaint.

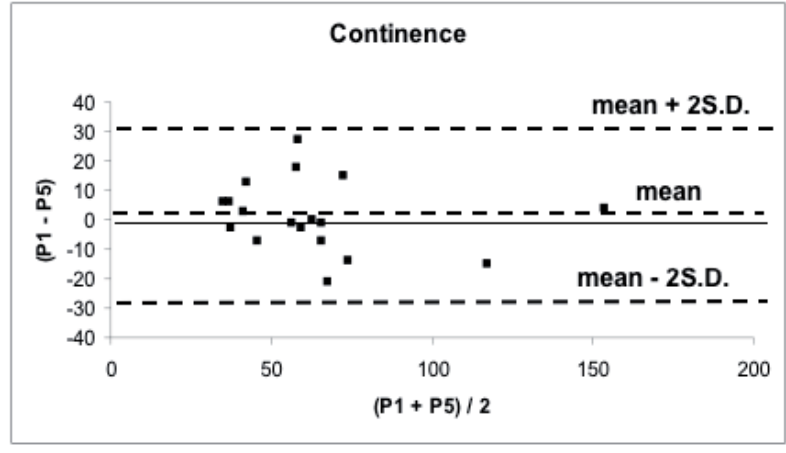

(a)

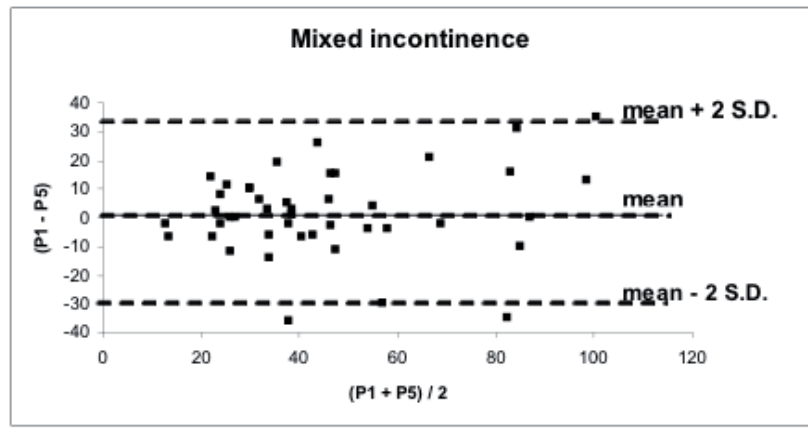

(c)

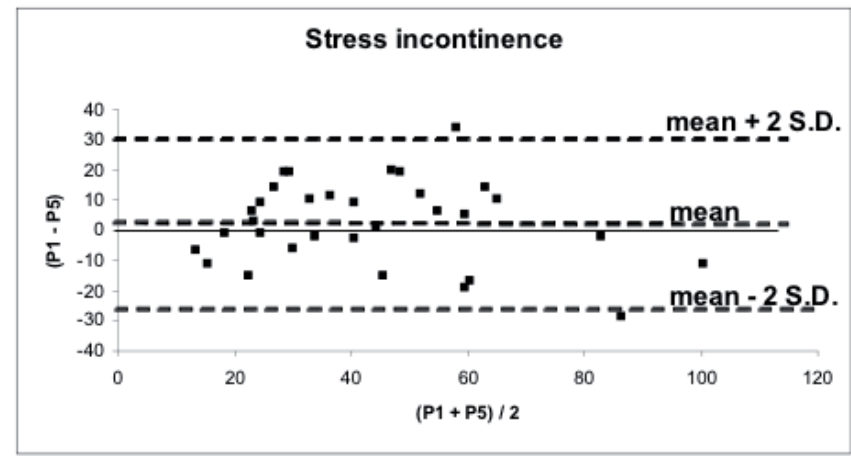

(b)

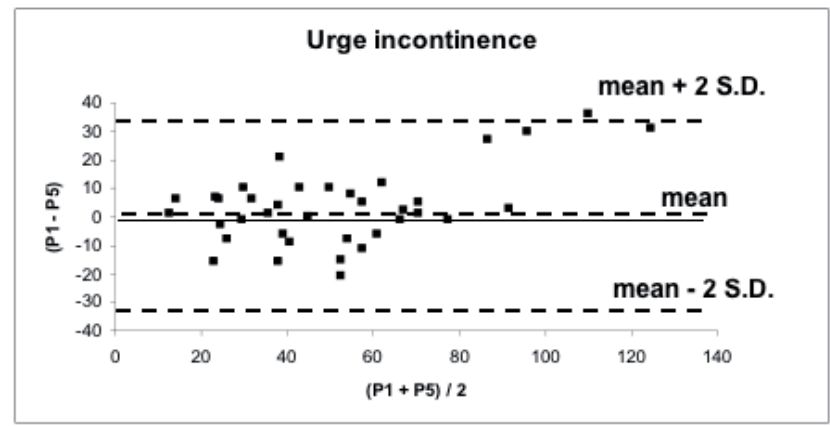

(d) 

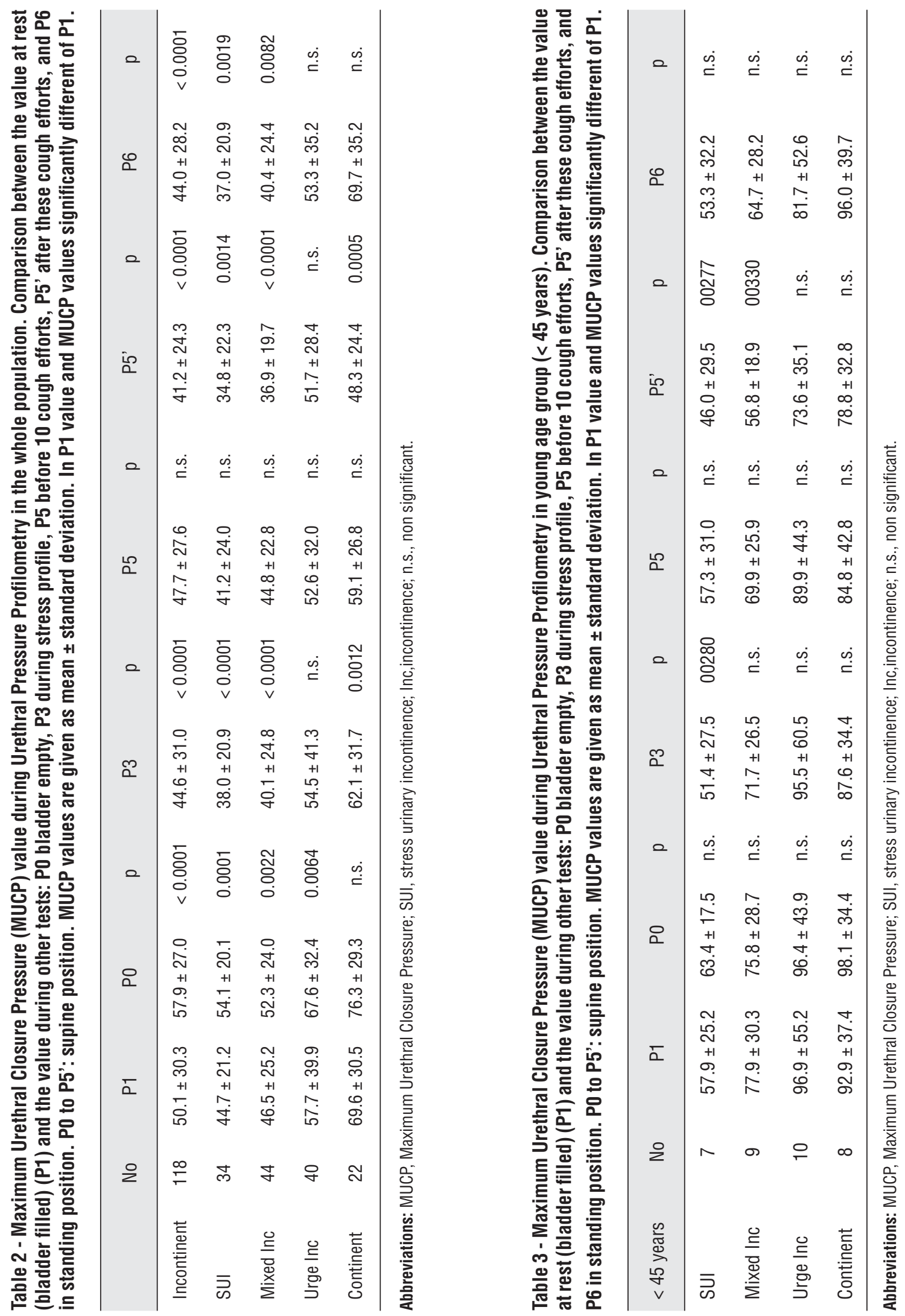


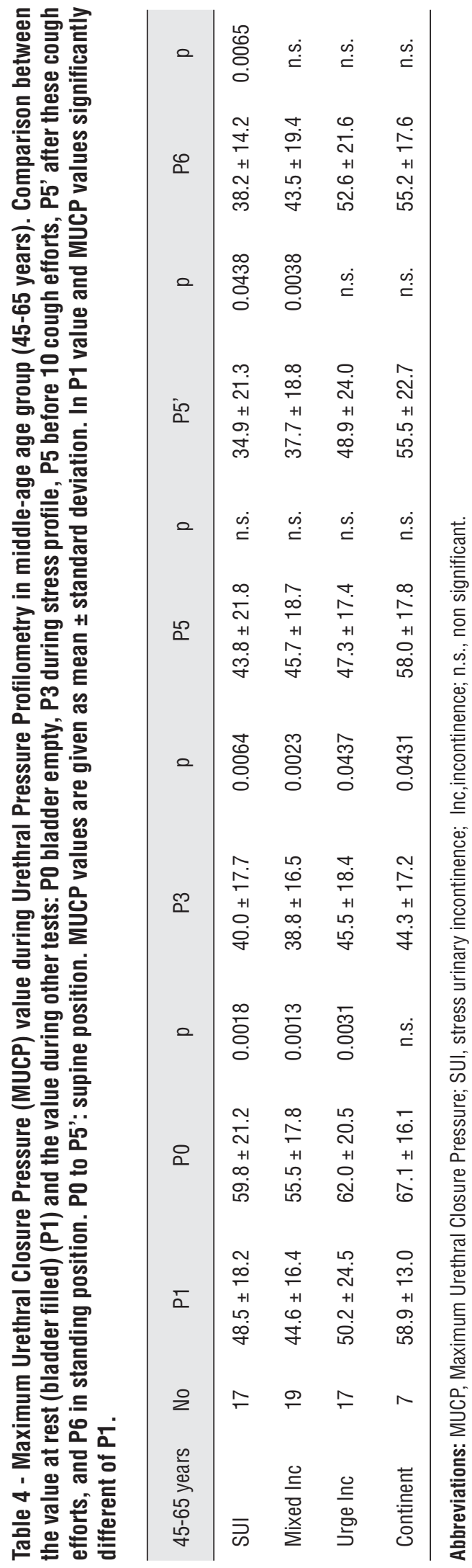

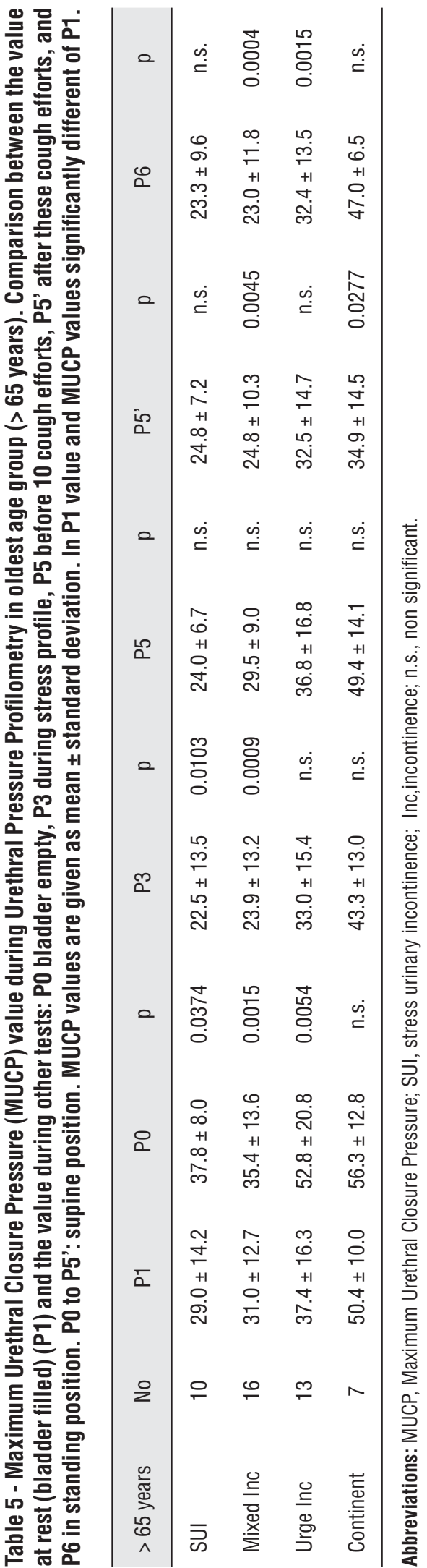




\section{DISCUSSION}

MUCP is a specific aspect of UPP. The main criticism addressed to it is that it is not an absolute measure of the urethral pressure, since it is an evaluation of the sphincter at rest, that does not provide information about the bladder neck or proximal urethra, that can be variable due to involuntary muscular contractions (irritant effect of the catheter), that the pressure varies with size and type of catheter, rate of perfusion, bladder volume and patient position (4), and that there is a wide variation in MUCP among individuals of similar age (16).

In relation to the causes of urinary incontinence in women, we must underline two urethral components: urethral hypermobility and intrinsic sphincter deficiency. It has been demonstrated that women with MUCP in the lowest quartile are likely to experience failure of mid urethral sling surgery (17) and that MUCP $\leq 40 \mathrm{~cm} \mathrm{H}_{2} 0$ is a risk factor affecting cure after transobturator tape procedure (5).

The conclusion of the ICS standardisation report on urethral pressure measurement (18) is that "the clinical utility of urethral pressure measurement is unclear" and that "there is no doubt that the urethral pressure is of significant importance for the continence mechanism".

Our study searched for the reproducibility of the MUCP value at rest, after filling the bladder, during a UPP session and tried to assess if different static and dynamic tests during that UPP session were correlated with the patient complaint.

During the UPP session, there was no significant change of MUCP value with filled bladder (P1 and P5) whatever the age and the complaint. That first result allowed us to use a complex UPP session and to search for a correlation between the change in MUCP value and the symptoms evoked before urodynamics.

Comparisons of MUCP value between full and empty bladder are still controversial: "some studies report lower mean MUCP with a full bladder, but others show no difference or even an increase in MUCP with bladder filling" (4). Most of these studies are limited to a comparison between "normal" and stress incontinent women without reference to the menopause status. In our population, MUCP value with empty bladder (P0) was almost always higher than MUCP with filled bladder (P1). However, the difference was only significant in incontinent women and, looking at age-groups, only in middle age and oldest subgroups. That variation can be explained by an impairment, occurring early with ageing, of both the sphincteric unit and the support system in women with stress or mixed incontinence; in women with urge incontinence that decrease can be the consequence of a voiding-like reflex mediated by changes in intercellular electrical coupling and localized contractions of smooth muscle due to the stretching of small portions of the bladder wall (19-20).

When we look at the specific condition of stress incontinence, resting MUCP value is known to tend to be lower than in continent women and decreases as a function of age (11-16), but there is no absolute cut-off value with sufficient sensitivity and specificity for that diagnosis and the association between MUCP and the severity of incontinence $(1,21,22)$. In our population, MUCP values at rest $(\mathrm{P} 1)$ tend to be lower in stress incontinent women except in the middle age group where women with mixed incontinence (which however implies a stress component) have lower MUCP.

When analyzing the results of the dynamic tests, we notice some differences. In standing position, looking at the whole population, a significant decrease of MUCP (P6) was only observed in women with stress or mixed incontinence which could be related to a deficiency of both intrinsic sphincter and peri-urethral muscles. In relation to the age groups, some differences appeared: the MUCP decreased only in women with SUI in the middle age and in women with mixed or urge incontinence in the oldest age. The previous assumption remains valid while the unexpected result for the oldest SUI group could be the consequence of a very low resting MUCP value. MUCP value remained unchanged in the continent group; that last result differs from the conclusion of Henriksson et al. (23) and Dörflinger et al. (24) but agrees with the conclusion of Lose (25).

In the whole population, MUCP decreased during the stress profile (P3) except in women with urge incontinence. In relation to age groups, 
that decrease occured always in SUI women, in all women in the middle age group and in women with stress or mixed incontinence in the oldest group. The decrease of MUCP during stress profile (P3) could be related to a urethral hypermobility as it has been demonstrated by Schick et al. (26). The difference between the middle age and the oldest group could be the consequence of a more rigid urethra due to oestrogen deficiency or pelvic surgery or both.

In the whole population, a MUCP decrease after the fatigability test (P5') was observed in the same groups as during stress profile (all except urge incontinence). In young and middle-age groups, a significant MUCP decrease was observed in women with SUI or mixed incontinence (stress component). In the oldest group, the non significant decrease of MUCP resulted from the lower resting value of MUCP as for standing position. The decrease of MUCP following repeated cough efforts (fatigability test) has been described in women with stress urinary incontinence (14) with the hypothesis of an increased fatigue of the peri-urethral muscles. In the whole population, we finded a similar decrease not only in women with stress incontinence but also in women with mixed incontinence due to the stress component.

Thus, this study showed that there was a good reproducibility of MUCP value at rest when a rigorous protocol of testing was applied. What can we conclude about a correlation between the patient complaints and the MUCP value change? In a first approach (i.e. the whole population) decrease of MUCP during dynamic testing was related to stress or mixed incontinence. Interestingly, that results must be cautiously analysed according to the age groups. In the youngest group (non menopausal women), the sequence of tests revealed the stress component of incontinence. In the middle-age group (peri-menopausal women) the sphincter behaviour was modified by the hormonal status and the fatigability test appeared to be the only dynamic test allowing to discriminate urge from stress. In the oldest group (long-term menopause), the low resting value of MUCP seemed to conceal most of the results of dynamic testing.

At last, a negative result (no MUCP decrease) during the fatigability test was observed wha- tever the age of women with urge incontinence complaint.

Criticism to our protocol could be that it is time consuming or that it can be exhausting. But in fact, our protocol only takes about $10 \mathrm{~min}$. and women do not complain of tiredness.

Limitations of this first study include the fact that this is a retrospective study and the stratifications of the population according with age (rough hormonal status) and symptoms (continence status) were not accurate; it would be completed by the approach of MUCP value changes according with the urodynamic diagnosis.

\section{CONCLUSIONS}

When a strict protocol is applied, MUCP at rest has a good reproducibility during a urethral profilometry session. Bladder filling induces a decrease in MUCP value in incontinent women whatever the type of incontinence in middle age and old age.

However, if a complex sequence of tests during urethral pressure profilometry remains discussed in peri- and long-term menopause women, it allows specifying the stress component of incontinence in non menopausal women and the urgency component in all incontinent women.

\section{ACKNOWLEDGEMENTS}

We thank Catherine Harchies and Odette Broussard, nurses in our laboratory, whom strict application of a rigorous protocol has allowed this study.

\section{CONFLICT OF INTEREST}

None declared.

\section{REFERENCES}

1. McGuire EJ, Fitzpatrick CC, Wan J, Bloom D, Sanvordenker J, Ritchey M: Clinical assessment of urethral sphincter function. J Urol. 1993; 150: 1452-4.

2. Lose G. Urethral pressure measurement. Acta Obstet Gynecol Scand. 1997; 166: 39-42. 
3. Duggan PM, Arnold EP: Assessment and management of female urinary incontinence--a survey of current practice. Aust N Z J Obstet Gynaecol. 1998; 38: 234-6.

4. Weber AM: Is urethral pressure profilometry a useful diagnostic test for stress urinary incontinence? Obstet Gynecol Surv. 2001; 56: 720-35.

5. Hsiao SM, Chang TC, Lin HH: Risk factors affecting cure after mid-urethral tape procedure for female urodynamic stress incontinence: comparison of retropubic and transobturator routes. Urology. 2009; 73: 981-6.

6. Costantini S, Nadalini C, Esposito F, Alessandri F, Valenzano MM, Mistrangelo E: Transobturator adjustable tape (TOA) in female stress urinary incontinence associated with low maximal urethral closure pressure. Arch Gynecol Obstet. 2010; 282: 277-84.

7. Lemack GE: Urodynamic assessment of patients with stress incontinence: how effective are urethral pressure profilometry and abdominal leak point pressures at case selection and predicting outcome? Curr Opin Urol. 2004; 14: 307-11.

8. Mikhail MS, Rosa H, Palan P, Anderson P: Comparison of preoperative and postoperative pressure transmission ratio and urethral pressure profilometry in patients with successful outcome following the vaginal wall patch sling technique. Neurourol Urodyn. 2005; 24: 31-4.

9. Wadie BS, El-Hefnawy AS: Urethral pressure measurement in stress incontinence: does it help? Int Urol Nephrol. 2009; 41: 491-5.

10. Drahorádová P, Masata J, Martan A, Svabík K, Pavlíková M: Can the preoperative value of VLPP and MUCP predict the postoperative quality of life? Prague Med Rep. 2009; 110: 42-50.

11. Delancey J0: Why do women have stress urinary incontinence? Neurourol Urodyn. 2010; 29(Suppl 1): S13-7.

12. Abrams $P$, Cardozo L, Fall M, Griffiths D, Rosier P, Ulmsten $U$, et al.: The standardisation of terminology of lower urinary tract function: report from the Standardisation Subcommittee of the International Continence Society. Neurourol Urodyn. 2002; 21: 167-78.

13. Schäfer W, Abrams P, Liao L, Mattiasson A, Pesce F, Spangberg $A$, et al.: Good urodynamic practices: uroflowmetry, filling cystometry, and pressure-flow studies. Neurourol Urodyn. 2002; 21: 261-74.

14. Deffieux X, Hubeaux K, Porcher R, Ismael SS, Raibaut $P$, Amarenco G: Decrease in urethral pressure following repeated cough efforts: a new concept for pathophysiology of stress urinary incontinence. Int J Urol. 2007; 14: 1019-24.

15. Bland JM, Altman DG: Statistical methods for assessing agreement between two methods of clinical measurement. Lancet. 1986; 1: 307-10.

16. Rud T: Urethral pressure profile in continent women from childhood to old age. Acta Obstet Gynecol Scand. 1980; 59: $331-5$.
17. Nager CW, Sirls L, Litman HJ, Richter H, Nygaard I, Chai T, et al.: Baseline urodynamic predictors of treatment failure 1 year after mid urethral sling surgery. J Urol. 2011; 186: 597-603.

18. Lose G, Griffiths D, Hosker G, Kulseng-Hanssen S, Perucchini $D$, Schäfer $W$, et al.: Standardisation of urethral pressure measurement: report from the Standardisation Sub-Committee of the International Continence Society. Neurourol Urodyn. 2002; 21: 258-60.

19. Brading AF: A myogenic basis for the overactive bladder. Urology. 1997; 50(6A Suppl): 57-67; discussion 68-73.

20. Elbadawi A, Yalla SV, Resnick NM: Structural basis of geriatric voiding dysfunction. III. Detrusor overactivity. J Urol. 1993; 150: 1668-80.

21. Hilton P, Stanton SL: Urethral pressure measurement by microtransducer: the results in symptom-free women and in those with genuine stress incontinence. Br J Obstet Gynaecol. 1983; 90: 919-33.

22. Theofrastous JP, Bump RC, Elser DM, Wyman JF, McClish DK: Correlation of urodynamic measures of urethral resistance with clinical measures of incontinence severity in women with pure genuine stress incontinence. The Continence Program for Women Research Group. Am J Obstet Gynecol. 1995; 173: 407-12; discussion 412-4.

23. Henriksson L, Ulmsten U, Andersson KE: The effect of changes of posture on the urethral closure pressure in healthy women. Scand J Urol Nephrol. 1977; 11: 201-6.

24. Dörflinger A, Gorton E, Stanton S, Dreher E: Urethral pressure profile: is it affected by position? Neurourol Urodyn. 2002; 21: 553-7.

25. Lose G: Impact of changes in posture and bladder filling on the mechanical properties of the urethra in healthy and stress incontinent females. Neurourol urodyn. 1990; 9: 459-69.

26. Schick E, Tessier J, Bertrand PE, Dupont C, Jolivet-Tremblay M: Observations on the function of the female urethra: I: relation between maximum urethral closure pressure at rest and urethral hypermobility. Neurourol Urodyn. 2003; 22: $643-7$ 